\title{
The STAR Silicon Drift Vertex Detector
}

Sanjeev Pandey ${ }^{a}$ and the STAR Collaboration ${ }^{*}$

${ }^{a}$ Wayne State University

The current status of the STAR Silicon Vertex Tracker (SVT) is presented. The performance of the Silicon Drift Detectors (SDD) is discussed. The enhanced physics capabilities of the STAR detector due to the addition of the SVT are addressed.

\section{Introduction}

A Silicon Drift Detector (SDD) is a charged particle position measuring device with a position resolution on the order of $20 \mu \mathrm{m}$ in each coordinate[1]. A charged particle crossing the detector creates electron-hole pairs. The holes are immediately absorbed by cathodes on the surface of the detector. Electrons converge to the middle of the bulk and drift at a constant speed under the influence of an applied uniform electric field toward low capacitance anodes located near the edge of the silicon wafer. One coordinate of the particle position is determined from the drift time while charge division between anodes gives the other coordinate.

Large $(6 \times 6 \mathrm{~cm})$ linear SDD have been developed and are in production[2] for use in the SVT, the inner tracking detector of the STAR experiment at the RHIC Collider[3-5]. The SVT consists of 216 wafers arranged in three concentric barrels at radii of $6.6,10.6$, and $14.5 \mathrm{~cm}$ respectively. The wafers are mounted on beryllium ladders and cover $|\eta|<1$ with $104 \mathrm{~K}$ readout channels and $0.7 \mathrm{~m}^{2}$ of silicon. The SVT resides in a $0.5 \mathrm{~T}$ magnetic field and is surrounded by a TPC.

The SVT project has been active for seven years and is one year from completion. It will significantly enhance the low transverse momentum (pT), strangeness, and event-byevent physics capabilities of the STAR detector. It allows measurements of yields and spectra for $\Lambda$ 's, $\Xi$ 's, $\Omega$ 's, and $K_{S}^{0}$ 's addressing questions about strangeness equilibrium and strangeness enhancement. It extends the low p'T range of the STAR detector to $60 \mathrm{MeV} / \mathrm{c}$ allowing questions concerning disoriented chiral condensates (DCC) to be addressed. It also enhances measurements of event-by-event particle ratios and pion HBT.

\section{The Silicon Vertex Tracker}

The SDD's have two symmetrical $3 \mathrm{~cm}$ long drift directions and 240 anodes spaced $250 \mu \mathrm{m}$ apart collect the signal at either end of the wafer as seen in fig 1 . The low capacitance anodes $(\approx 200 \mathrm{fF})$ are matched with low noise frontend electronics (FEE) leading to a combined system noise of about 530e[6]. A minimum ionizing particle deposits $24000 \mathrm{e}$ 


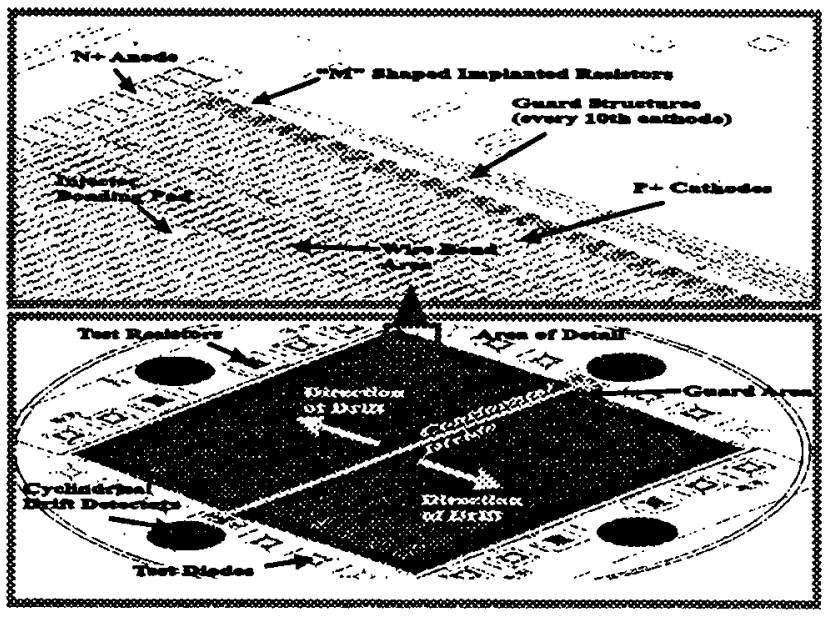

Figure 1. A STAR-SVT bi-linear Silicon Drift Detector.
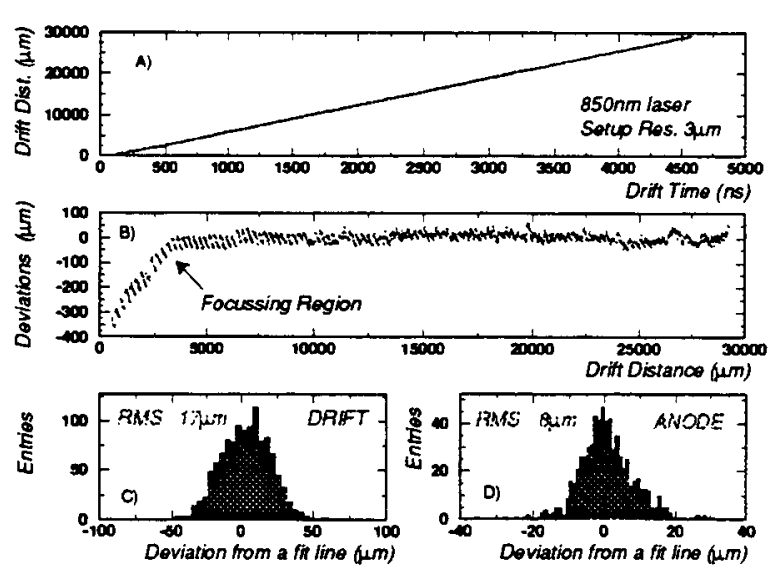

Figure 2. A) Drift linearity on a typical detector. B) Deviations from a linear fit to Figure A. C) and D) Projections of Figure $\mathrm{B}$ on the $\mathrm{Y}$ axis ignoring the focussing region.

in the SDD. A drift potential of $500 \mathrm{~V} / \mathrm{cm}$ is applied and at room temperature electrons in the wafer drift at $6.6 \mu \mathrm{m} / \mathrm{ns}$. The total drift time is $4.5 \mu \mathrm{s}$ and corresponds to the dead time of the detector. The drift direction is sampled via a switched capacitor array[7] that records 128 time samples at a $25 \mathrm{MHz}$ clock frequency effectively creating a pixel-like detector with $250 \times 240 \mu \mathrm{m}$ pixels. This pixel-like readout allows high density tracking with a low number of readout channels. The average pixel occupancy in the detector is $<2 \%$. It takes $5 \mathrm{~ms}$ to readout all 216 wafers in the SVT. Assuming 2500 charged tracks per event, 24 pixels per hit, and 1 byte per pixel, one records 180KB (2500 X 24 X 3 layers) for a zero-suppressed SVT event.

Presently, position resolution is determined in the laboratory by mounting a laser $(850 \mathrm{~nm})$ on an XY stage and creating a MIP signal while scanning across the detector surface. Results from a typical detector are shown in fig. 2 where a position resolution below $20 \mu \mathrm{m}$ is measured. Resolution along the drift direction is worse than along the anode due to nonlinearities in the drift field caused by non-homogeneous implanted resistors. The SDD measures the charge deposited for each hit allowing $\mathrm{dE} / \mathrm{dx}$ measurements for particle identification as seen in fig. 3a. The average $\mathrm{dE} / \mathrm{dx}$ resolution in the three layer SVT is $7 \%$ for a MIP particle. Results from a 15 SDD plane prototype in experiment E896 using the same FEE and readout electronics as the SVT show similar behavior $[8,9]$. Within measuring accuracy there is no evidence of charge loss at large drift distances as seen in fig. 3b. The peak amplitude decreases with increasing drift distance in fig. 3c due to diffusion. This impacts the two track resolution of the detector which is proportional to the cloud width and is $500 \mu \mathrm{m}$ at longest drift.

The SDD's response is sensitive to certain environmental conditions such as the drift electrical field, the temperature, and the external magnetic field $[10,11]$. The drift velocity 

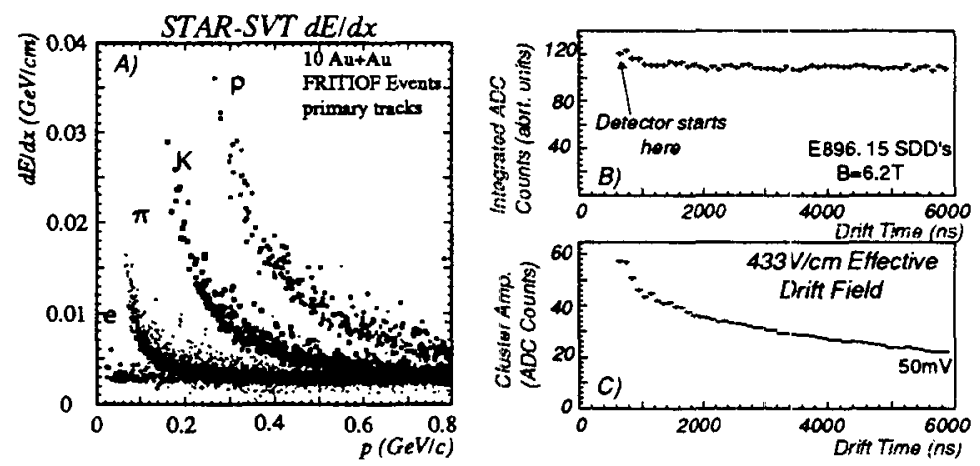

Figure 3. A) The expected $d E / d x$ for tracks in the STAR SV'T. B) The integrated charge of hits in a SDD vs. drift distance. C) The decrease in amplitude for these hits with drift distance. The SVT will run at $500 \mathrm{~V} / \mathrm{cm}$ which increases the peak amplitude.

changes by $1 \%$ for each Kelvin increase in temperature and thus the SVT environment will be controlled to a maximum $0.5 \mathrm{~K}$ fluctuation. Changes in temperature are monitored by changes in drift time through MOS charge injector lines on the SDD surface.

Over $70 \%$ of the detectors and $60 \%$ of the hybrid electronics exist. Commercial production of the SSD was very successfull resulting in a $70 \%$ yie.ld for the first 300 produced wafers. Assembly of ladders holding the detectors and associated electronics has commenced. A full sector of the SVT is being built and testing with the associated slow controls, readout electronics, and DAQ has started. The position of all components on the sector are know to within $20 \mu \mathrm{m}$. The SVT has a radiation length of $1.4 \% /$ layer $(0.3 \%$ Silicon $+0.5 \%$ FEE $+0.6 \%$ water cooling). A clam-shell detector support structure consisting of beryllium detector carriers and carbon fiber electronic carriers that allow water cooling is being constructed. The SVT support cone is already installed and supports the STAR beryllium beam pipe. The SVT will be ready for installation in the summer of 2000 .

\section{Physics Enhancement}

The SVT and the SSD, a 4th layer silicon strip detector[12], increase the acceptance and reconstruction efficiency for weakly decaying strange particles as seen in fig. 4 . The SVT/SSD will allow a more accurate measurement of the main vertex allowing close secondary vertices to be resolved as well as allowing secondary vertices to decay within the SVT volume. The improved low pT tracking, down to $60 \mathrm{MeV} / \mathrm{c}$, helps reconstruct the low momentum pion from the lambda as well as allowing DCC analysis to be explored. The SVT will allow $K_{S}^{0} K_{S}^{0}$ interferometry and might allow event by event HBT for small source sizes depending on the multiplicity of the events. 


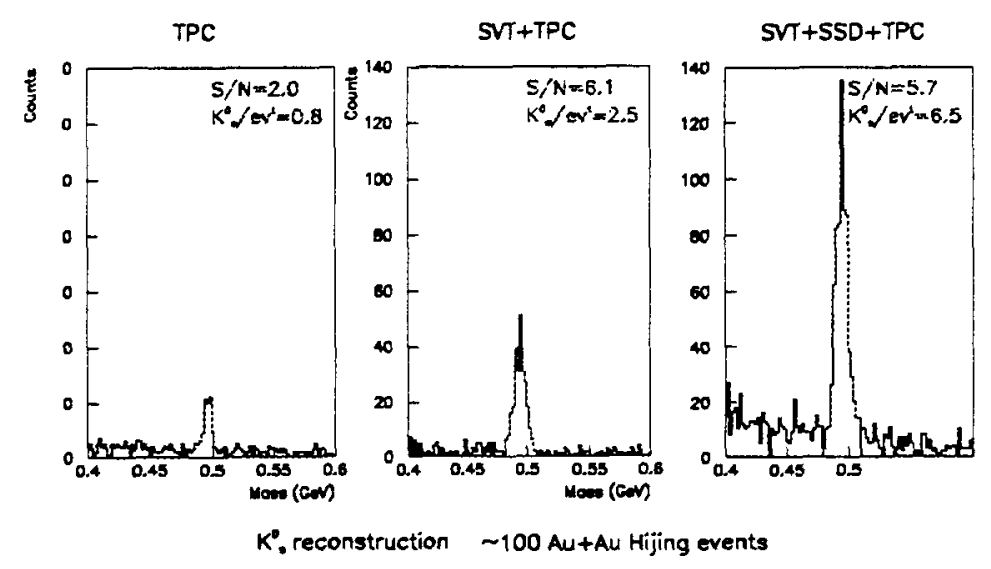

Figure 4 . The $K_{S}^{0}$ reconstruction efficiency for STAR with different detector subsystems.

\section{Conclusions and Acknowledgments}

The STAR SVT is ready for installation in the summer of 2000 . The first tracking device based on silicon drift detectors has been built and operated successfully using SVT prototype wafers, frontend electronics and readout electronics in AGS experiment E896. The detectors perform well. The technology for making and using large silicon drift detectors as tracking devices has now matured to a large production level.

This work was supported in part by the U.S. Department of Energy under contract DE-AC03-76SF00098.

\section{REFERENCES}

1. E Gatti and P Rehak, Nucl. Instr. and Meth., vol. 225, (1984) pp. 608.

2. J Takahashi et al, 8th European Symposium of Semiconductor Det., (1998) Submitted.

3. J W Harris et al, Nucl. Phys., vol. A566, (1994) pp. 277c.

4. H Weiman et al, Nucl. Phys., vol. A566, (1994) pp. 277c.

5. J Thomas et al, Parallel session report in these proceedings.

6. D Lynn et al, 8th European Symposium of Semiconductor Det., (1998) Submitted.

7. S A Kleinfelder, IEEE Transactions on Nuclear Science, vol. 37, (1990) pp. 1230.

8. G Lo Curto et al, Parallel session report in these proceedings.

9. H Caines et al, Plenary session report in these proceedings.

10. S U Pandey et al, Nucl. Instr. and Meth., vol. A383, (1996) pp. 537.

11. R Bellwied et al, Nucl. Instr. and Meth., vol. A377, (1996) pp. 387.

12. A Boucham,et al, STAR Collaboration Note 400, (1999) 